\title{
REMARQUES SUR LE GENRE DINOBOTHRIUM VAN BENEDEN 1889
}

(CESTODA-TETRAPHYLLIDEA)

\author{
Par Louis EUZET
}

\section{Historique}

C'est en 1889 que Van Beneden décrivit « Deux Cestodes nouveaux de Lamna cornubica ». L'un de ces parasites est nommé Dinobothrium septaria n. g., n. sp., l'autre Diplobothrium simile n. g., n. sp. N'ayant pu examiner que quelques individus fixés, l'auteur belge donne seulement des renseignements sur l'anatomie externe de ces deux formes. En 1892, Lönnberg récolta chez Lamna cornubica des parasites qui correspondaient à ceux de Van Beneden. Il décrivit alors avec assez de détails le scolex de Dinobothrium septaria. En 1897, il étudia, provenant toujours du même hôte (Isurus cornubicus), des vers qu'il rapporta aux deux genres cités ci-dessus. Il figura même l'anatomie interne, semblable dans les deux formes, surtout remarquable par la course antérieure du vagin.

Mola, en 1906, recueillit en Méditerranée, chez Selache maxima (Cetorhinus maximus Gunner), des strobiles de 80 à $120 \mathrm{~mm}$. qu il nomma Dinobothrium septaria et dont il donna un schéma de l'anatomie. Celle-ci se caractérisait par la disposition des vitellogènes en une seule couche ventrale.

En 1908, Scott obtint un spécimen de Dinobothrium septaria chez Lamna cornubica pêché en Mer du Nord.

Masi, en 1912, récolta un parasite chez Selache maxima en Méditerranée.

En 1914, Nybelin étudia les diverses allures du scolex de spécimens récoltés chez Selache maxima, cestodes qu'il rapporta à Dinobothrium septaria.

Linton, en 1922, publie une note sur le genre Dinobothrium, où il étudie d'une part le scolex de $D$. plicitum n. sp., espèce immature récoltée dans l'intestin de Carcharodon carcharias, et d'autre part le scolex et l'anatomie de Dinobothrium planum n. sp. Cette deuxiè-

Ann. de Parasitologie, T. XXX, $\mathrm{N}^{\circ} 3 .-1955$. 
me espèce provenant de Cetorhinus maximus est totalement différente de $D$. septaria. Son anatomie, en particulier, se distingue par les vitellogènes folliculaires disposés en une seule couche ventrale.

Joyeux, en 1923 , rapporte à $D$. plicitum des parasites trouvés dans la valvule spirale d'un Pèlerin (Cetorhinus maximus), à Concarneau (Finistère).

Southwell, dans sa monographie de 1925, classe ces Cestodes dans le genre Phyllobothrium avec une seule espèce, $P$. septaria (Van Ben. 1889).

C'est contre cette manière de voir que s'élève Woodland lorsque, en 1927, il reprend l'étude anatomique de l'espèce de Van Beneden. Ces Cestodes avaient été trouvés dans la partie postérieure de l'intestin spiral d'un jeune Lamna cornubica (Plymouth, Octobre 1925). L'auteur conclut à la validité du genre et à la distinction de deux espèces : D. septaria Van Ben. 1889 et D. planum Linton 1922. Woodland, mettant en doute les observations de Mola et Linton sur les vitellogènes, base sa différenciation sur des caractères externes. Pour l'anatomie, il les sépare par la forme et la taille du vagin et de la poche du cirre. L'espèce $D$. plicitum est pour lui considérée comme synonyme de $D$. septaria.

Perrenoud, en 1931, reprend l'étude anatomique de D. septaria avec le matériel récolté par R. Legendre à Concarneau. Sa description est, à quelques détails près, semblable à celle de Woodland et ses conclusions sont identiques.

Tseng, en 1933, représente sous le nom de Dinobothrium septaria un Cestode récolté dans la valvule spirale d'un Cetorhinus maximus à Chefoo-Shantung (Chine).

Dans La Faune de France, Joyeux et Baer, en 1936, ne signalent que D. septaria, mais placent Cetorhinus maximus à côté de Lamna cornubica dans la liste des hôtes.

En 1945, D. Guevara Pozo étudie sous le nom de D. planum des parasites d'un Cetorhinus maximus capturé à Palma de Mayorque, et décrit comme $D$. plicitum des spécimens immatures récoltés chez ce même hôte.

Miss N. Sproston reprit la question dans son travail de 1948. Après avoir étudié $D$. septaria et $D$. planum, elle décrit deux espèces nouvelles, à savoir : $D$. keilini de Carcharhinus glaucus et $D$. paciferum de Cetorhinus maximus.

En 1950, Baylis fait une révision eritique des espèces du genre et décrit une nouvelle espèce. Dinobothrium spinosum de Cetorhinus maximus.

C'est dans cet hôte que Yamaguti retrouve ce Cestode et, n'ayant pas connaissance du travail de Baylis, le nomme Dinobothrium spi- 
nulosum n. sp. Il retrouve aussi D. planum, mais, tenant compte de l'anatomie très particulière de ce ver, le place dans un nouveau genre qu'il nomme Gastrolecithus.

Wardle et Mc Leod, en 1952, n'apportent aucun argument intéressant à la question.

Rappelons que nous avons nous-même décrit, toujours en 1952, d'un Cetorhinus pêché à Naples, un Dinobothrium humile que nous avons assimilé à Dinobothrium spinosum Baylis 1950 .

Rappelons encore que nous avons, avec C. Delamare-Debouteville, retrouvé et signalé ce $D$. spinosum avec $D$. planum chez Cetorhinus maximus (Cabanes-de-Fleury, Aude, 12 mai 1952).

Voilà l'histoire de ce genre qui, nous le verrons, est un des plus particuliers parmi les Cestodes Tétraphyllides.

$$
\text { * * } *
$$

Après examen du matériel original de N. Sproston et de Baylis (matériel déposé au British Muséum), l'étude de nombreux exemplaires de la Collection Dollfus (recueillis à Concarneau par luimême et par R. Legendre), l'examen des individus de la Collection I.F.A.N. (récoltés par J. Cadenat), de ceux de la Collection de l'Université de Neuchâtel (J.-G. Baer) et des individus que nous avons personnellement collectés, nous pensons que seules trois espèces sont valables parce qu'elles sont bien caractérisées.

Ces trois espèces sont :

Dinobothrium septaria Van Ben. 1889 ;

Dinobothrium planum Linton 1922 ;

Dinobothrium paciferum Sproston 1948.

Une espèce reste douteuse, c'est le Dinobothrium plicitum Linton 1922. Le ver était immature et, la différence entre les diverses espèces étant surtout basée sur l'anatomie, il ne peut être mis en synonymie avec l'une ou l'autre. Seule, la récolte chez Carcharodon carcharias d'individus adultes pourrait trancher la question.

Une autre question se pose tout d'abord. Dans son mémoire original, Van Beneden décrit deux Cestodes. Est-ce que ces deux vers, Dinobothrium septaria et Diplobothrium simile, sont deux genres et espèces différents ou bien une seule et même espèce ?

Van Beneden a basé la différence sur la taille du ver et surtout sur la forme du scolex ; mais il n'a pas étudié l'anatomie, les strobiles étant trop peu développés et trop mal conservés.

Baylis pense que le matériel original de Van Beneden était de la 
même espèce, mais, les Cestodes étant immatures, la question ne saurait être tranchée par l'anatomie. Cet auteur écrit que Van Beneden, Lönnberg et Scott ont vu des scolex dans des états de contraction extrême ou très relâchés. Mais il lui paraît étrange que Lönnberg ait pu donner des figures identiques de l'anatomie de deux vers et ait pu les croire différents, non seulement spécifiquement, mais encore génériquement, et ce à cause des aspects différents du scolex.

Après le travail de Nybelin sur les allures diverses de ce scolex selon le mode de fixation, après l'étude et les figures de Perrenoud et après nos propres observations, nous sommes persuadés que Van Beneden n'a eu affaire qu'à une seule et même espèce. Ceci est confirmé par le fait que Lönnberg a décrit sous deux noms des Cestodes dont l'anatomie est identique. Nous suivons donc Joyeux et Baer et considérons Diplobothrium simile comme synonyme de Dinobothrium septaria.

Avant de pouvoir conclure sur ce genre particulier, nous devons donner une étude morphologique et anatomique des espèces que nous reconnaissons comme valables :

\section{DINOBOTHRIUM SEPTARIA Van Ben. 1889}

Syn. Diplobothrium simile Van Ben. 1889.

Dinobothrium septaria Lönnberg 1892-1898, hôte Lamna cornubica. Dinobothrium septaria Scott 1909, hôte Lamna cornubica.

Phyllobothrium septaria Southwell 1925 (partim), hôte Lamna cornubica.

Dinobothrium septaria Woodland 1927, hôte Lamna cornubica.

Dinobothrium septaria Perrenoud 1931, hôte Lamna cornubica.

Dinobothrium septaria Joyeux et Baer 1936 (partim), hôte Lamna cornubica.

Dinobothrium septaria Sproston 1948, hôte Lamna cornubica.

Dinobothrium keilini Sproston 1948, hôte Carcharhinus glancus.

Dinobothrium septaria Baylis 1950, hôte Lamna cornubica.

Dinobothrium septaria Wardle et Mc. Leod 1952, hôte Lamna cornubica.

nec. Dinobothrium septaria Mola 1907, Masi 1912, Nybelin 1914, Tseng 1933.

Matériel étudié :

Dinobothrium septaria Woodland et Perrenoud (coupes).

Dinobothrium keilini, types de Sproston.

Dinobothrium septaria, Lamna cornubica, Concarneau, 19-9-1927 (coll. R.-Ph. Dollfus). 
Comme nous l'avons écrit ci-dessus, nous pensons avec Baylis que l'espèce de N. Sproston, D. keilini, est semblable à celle de Van Beneden. Les différences énoncées ne sont pas suffisantes pour séparer les deux espèces. Cependant, l'hôte Carcharhinus glaucus est inhabituel. Ce parasite doit être rare dans ce Sélacien, car nous ne l'avons jamais rencontré, ni dans les divers matériaux, provenant de cet hôte, que nous avons examinés, ni durant nos propres recherches.

Nous sommes d'accord avec Sproston et Baylis sur le fait que le nom de $D$. septaria doit seul s'appliquer aux Dinobothrium de Lamna cornubica et de Carcharhinus.

Les Cestodes décrits sous ce nom chez Cetorhinus maximus doivent être rapportés à Dinobothrium planum Linton 1922.

\section{Morphologie}

Les individus de Van Beneden mesuraient de 25 à $30 \mathrm{~mm}$. Ceux que nous avons étudiés ont de 60 à $80 \mathrm{~mm}$. de long. Ces valeurs sont voisines de celles données par Woodland : $110 \mathrm{~mm}$., et Perrenoud : 70 à $80 \mathrm{~mm}$., mais certains auteurs ont signalé des individus non mûrs mesurant jusqu'à $180 \mathrm{~mm}$. La largeur varie de 5 à $6 \mathrm{~mm}$.

Le scolex se présente sous deux aspects différents que Nybelin a nettement caractérisés pour D. planum. Le type A (fig. 1) a des bothridies retombantes, c'est-à-dire parallèles aux faces dorsale et ventrale du ver, tandis que, dans le type B (fig. 2), les quatre bothridies globuleuses s'ouvrent perpendiculairement à l'axe du ver. Nybelin pense que le type A est réalisé lorsque le ver est fixé dans les replis de la valvule spirale (deux bothridies s'appuient sur une lame, les deux autres sur la lame immédiatement supérieure), tandis que le type B, qui ne présente qu'une surface d'adhésion formée par quatre bothridies dirigées en avant, est l'aspect du ver fixé à une seule paroi de l'intestin.

Chez les exemplaires que nous avons examinés, les scolex du type B mesuraient $3 \mathrm{~mm}$. de long et $5 \mathrm{~mm}$. de large, dimensions voisines de celles données par Perrenoud qui a eu un matériel de même type. Par contre, chez D. keilini de type A, le scolex mesurait sur le vivant $8,3 \mathrm{~mm}$. de long sur $10 \mathrm{~mm}$. de large (Sproston).

Les bothridies $(8 \mathrm{~mm}$. $\times 5 \mathrm{~mm}$. environ) sont en forme de cuiller et réunies en paire dorsale ou ventrale. Très musculeuses, elles représentent de puissants moyens de fixation. Leur surface convexe est recouverte de nombreuses petites épines. Chaque bothridie est surmontée d'un plateau musculaire, formant à chaque extrémité 
une pointe mousse. Du côté distal (externe), le plateau se prolonge sur la face dorsale de la bothridie par un lobe divisé en deux pointes. Au tiers du bourrelet, du côté axial, s'ouvre une petite ventouse accessoire de $350 \mu$ de diamètre. "Cette ventouse, écrit Van Beneden, dont on ne voit que la moitié, est collée à une portion saillante comme le nid de l'hirondelle Salangane est collé au rocher. »

Le scolex est porté par un cou très court.

Les segments, nous en avons compté une centaine d'abord comme un simple trait, s'allongent très lentement pour devenir chez les individus contractés à peine aussi longs que larges $(3 \mathrm{~mm} . \times$ $3 \mathrm{~mm}$.). Chez d'autres individus, on a noté que ces derniers segments étaient environ deux fois plus longs que larges.

Le pore sexuel alterne irrégulièrement et s'ouvre, latéralement, entre la $1 / 2$ et le $1 / 3$ inférieur du segment.

\section{Anatomie (fig. 3)}

ApPareil MÂle. - Les testicules sont très nombreux et s'étendent dorsalement en deux champs latéraux entre l'ovaire et les vitellogènes. Leur nombre varie entre 600 et 650 .

Perrenoud a donné de 320 à 400 testicules, et Baylis en a compté 750 chez un jeune segment à peine mûr de Dinobothrium keilini. Compte tenu des erreurs possibles (Perrenoud donne ses chiffres d'après reconstitution sur coupes), nous pensons que ces différences peuvent entrer dans l'ordre de variations spécifiques.

Le canal déférent décrit des sinuosités groupées en peloton au centre du segment, à l'extrémité distale de la poche du cirre. Celleci, énorme ( 1 à $1,4 \mathrm{~mm}$. $\times 0,45$ à $0,5 \mathrm{~mm}$.), contient un très long pénis armé d'épines. Ce cirre, élargi à la base, est couvert dans cette partie d'épines de 35 à $40 \mu$ (fig. 4 ).

Ces dernières, qui vont en diminuant de taille vers l'extrémité distale, ne mesurent plus que 25 à $30 \mu$ au milieu et 6 à $8 \mu$ à l'extrémité. Entre les épines, on distingue de nombreuses soies. Le cirre évaginé mesure de 8 à $10 \mathrm{~mm}$. de long.

Appareil femelle. - L'ovaire s'étend dans le $1 / 5$ inférieur de l'anneau. Il est tétralobé et a, en coupe, l'allure en $\mathrm{X}$ donnée par Woodland, comme caractéristique des Phyllobothriidæ (fig. 5).

Le vagin débouche antérieurement à la poche du cirre. Il se caractérise par une partie ascendante marquée par deux puissants muscles sphincter, un près de l'ouverture génitale, l'autre au milieu de la course. De ce sphincter, à la partie antérieure, le vagin forme de nombreux replis, puis descend vers le pont ovarien par un trajet 

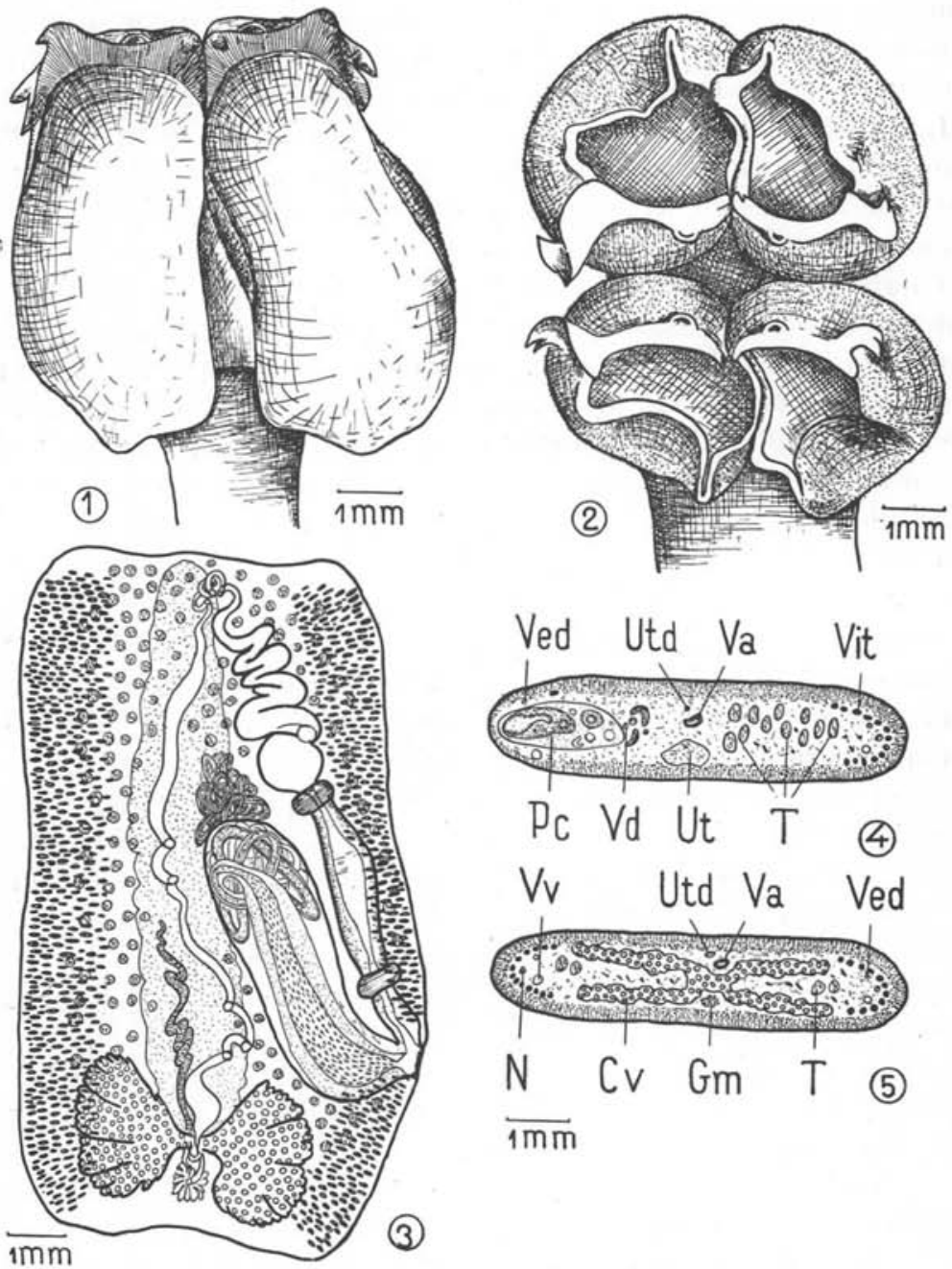

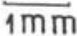

FIG. 1. - Dinobothrium septaria VAN BENEDEN, 1889.

Scolex forme A à bothridies retombantes. Vue ventrale.

Fig. 2. - Dinobothrium septaria VAN Beneden, 1889.

Scolex forme B à bothridies globuleuses. Vue apicale.

Fin. 3. - Dinobothrium septaria VAN Beneden, 1889.

Segment sexué, préparation totale.

Fic. 4. - Dinobothrium septaria VAN Beneden, 1889.

Coupe au niveau de la poche du cirre.

Fig. 5. - Dinobothrium septaria Van Beneden, 1889. Coupe au niveau de l'ovaire. Gm, glande de Mehlis ; N, nerf ; Ov, ovaire ; Pc, poche du cirre ; T, testicule ; Ut, utérus ; Utd, utéroducte ; Va, vagin ; Ved, vaisseau excréteur dorsal ; Vev, vaisseau excréteur ventral ; Vd, vas deferens ; Vit, vitellogènes. 
sinueux dans la ligne médiane de l'anneau; juste avant le pont ovarien, il se renfle en un court réceptacle séminal et débouche par un canal à lumière très étroite dans l'oviducte.

Les vitellogènes folliculaires forment deux bandes latérales, interrompues seulement au niveau de l'ouverture génitale. Ils s'étendent sur les faces ventrale et dorsale de l'anneau et ont en coupe un aspect en $\mathrm{U}$.

La glande de Mehlis est située entre les lobes de l'ovaire à l'extrémité postérieure du proglottis.

L'oviscape en tronc de cône large $(70 \mu)$ est bien marqué. Il se continue par l'oviducte qui, après avoir reçu le vagin, forme une boucle dans la glande de Mehlis, au niveau de laquelle il reçoit le canal vitellin. Il remonte ensuite et, après un trajet en $\mathrm{S}$, conduit par un utéroducte sinueux dans l'utérus. Ce dernier se présente d'abord comme une bande médiane ventrale de cellules différenciées. Dans les segments gravides, il occupe tout le centre du segment avec un petit nombre d'importantes expansions latérales.

Les œufs sphériques ont environ $25 \mu$ de diamètre.

Le système excréteur est typiquement représenté dans le strobile par quatre canaux longitudinaux (de chaque côté, un canal ventral large et un canal dorsal étroit).

\section{GASTROLECITHUS PLANUS (Linton 1922)}

Syn. Dinobothrium septaria Mola 1907, hôte Cetorhinus maximus. Dinobotthrium septaria Masi 1912, hôte Cetorhinus maximus.

Dinobothrium septaria Nybelin 1914, hôte Cetorhinus maximus.

Dinobothrium planum Linton 1922, hôte Cetorhinus maximus.

Dinobothrium plicitum Joyeux 1923, hôte Cetorhinus maximus.

Phyllobothrium septaria Southwell 1925 (partim), hôte Cetorhinus maximus.

Dinobothrium septaria Tseng 1933, hôte Cetorhinus maximus.

Dinobothrium planum Guevara Pozo 1945, hôte Cetorhinus maximus.

Dinobothrium septaria Joyeux et Baer 1936 (partim), Cetorhinus maximus.

Dinobothrium planum Sproston 1948, hòte Cetorhinus maximus.

Dinobothrium planum Baylis 1950, hôte Cetorhinus maximus.

Dinobothrium planum Wardle et Mc. Leod 1952, hôte Cetorhinus maximus.

Gastrolecithus planus Yamaguti 1952, hôte Cetorhinus maximus.

Dinobothrium planum Delamare et Euzet 1952, hôte Cetorhinus maximus. 
Comme nous l'avons écrit à propos de $D$. septaria, nous pensons que le nom de Gastrolecithus planus doit s'appliquer aux Dinobothrium de grande taille trouvés dans la valvule spirale de Cetorhinus maximus.

L'anatomie est, comme nous allons le voir, très particulière. Il s'ensuit que des auteurs comme Woodland, et Perrenoud, qui eux étudiaient $D$. septaria, ont pu mettre en doute les observations très précises de Mola et Linton et même les traiter d'erronées.

\section{Matériel étudié :}

Dinobothrium planum, matériel et coupe de Baylis.

Gastrolecithus planus, Cetorhinus maximus, Concarneau, 18-7-1938. Gastrolecithus planus, Cetorhinus maximus, Concarneau, 9-4-1943. Gastrolecithus planus, Cetorhinus maximus, Concarneau, 6-4-1943. (Collection R.-Ph. Dollfus)

Gastrolecithus planus, Cetorhinus maximus Hann (Dakar), 2324, 11-46 (Collection I.F.A.N., J. Cadenat collect.)

Gastrolecithus planus, Cetorhinus maximus Cabanes de Fleury (Aude), 12-5-1952.

\section{Morphologie}

Ver anapolytique très variable dans sa taille. On a signalé des individus mesurant $120 \mathrm{~mm}$. et d'autres $825 \mathrm{~mm}$. de longueur. Les exemplaires adultes que nous avons récoltés avaient respectivement 280,220 et $210 \mathrm{~mm}$. de long.

La largeur est aussi très variable. Elle était de 3 à $4 \mathrm{~mm}$. chez les segments gravides de nos individus, mais elle peut atteindre $7 \mathrm{~mm}$. (Yamaguti).

Nous avons compté 550 segments chez un individu de $280 \mathrm{~mm}$. Le scolex est porté par un cou ou portion non segmentée de $40 \mathrm{~mm}$. Les segments, d'abord comme un simple trait transverse, s'allongent très lentement. Dans les exemplaires examinés, aucun segment n'est plus long que large.

Le scolex se présente, comme D. septaria (Nybelin 1914), sous deux formes. Chez nos individus, il mesurait $8 \mathrm{~mm}$. de large et $6,5 \mathrm{~mm}$. de long. Les bothridies en cuiller mesurent $6,5 \mathrm{~mm}$. de long et $4 \mathrm{~mm}$. de large. Leur dos est recouvert de petites épines et leur bord postérieur parfois plissé suivant l'axe médian. L'allure est alors celle que Linton a dessinée pour $D$. plicitum. Chaque bothridie est surmontée d'un bourrelet musculaire qui présente deux petites pointes à chaque extrémité. Ces pointes sont plus ou moins visibles selon l'état de contraction du scolex. 
Du côté externe, le bourrelet se prolonge dans le dos de la bothridie par un lobe à deux pointes (fig. 6).

A la face apicale du bourrelet s'ouvre une petite ventouse en demi-cercle. Cette ventouse qui mesure $500 \mu$ environ de diamètre, si elle n'est pas complète, semble mieux formée que chez $D$. septaria.

Les bothridies sont reliées dorsalement par paire ventrale et dorsale.

Le strobile est linéaire, mais lors de la fixation il a tendance à s'enrouler en tire-bouchon. Il est nettement reconnaissable à un sillon médian qui s'étend sur les faces ventrale et dorsale. Ces sillons, mieux marqués sur la face ventrale, sont invisibles dans la partie non segmentée et ont tendance à s'atténuer chez les segments gravides.

Le pore sexuel alterne irrégulièrement et s'ouvre latéralement au milieu ou dans le deuxième $1 / 4$ antérieur de l'anneau.

\section{Anatomie (fig. 7)}

APPAREIL mâle. - Les testicules sont nombreux, nous en avons compté de 250 à 300 par segment. Ils sont groupés en deux bandes dorsales qui s'étendent postérieurement de chaque côté de l'ovaire. Le canal déférent forme un peloton allongé, qui va du milieu du proglottis à l'extrémité de la poche du cirre. Celle-ci, sub-cylindrique, parfois enroulée, mesure de 600 à $650 \mu$ de long et de 150 à $175 \mu$ de large (fig. 8). Elle contient un pénis court, armé d'épines droites, minces de $15 \mu$.

Appareil femelle. - Le vagin s'ouvre dans le pore génital commun antérieurement à la poche du cirre, mais parfois à côté et ventralement à cette dernière.

Il se dirige horizontalement par un trajet très sinueux jusqu'à la ligne médiane du segment, où il forme un coude et descend vers le complexe ovarien. Il se rétrécit alors et se jette dans l'oviducte, tout près de l'oviscape.

Cet oviducte fait un coude dans la glande de Mehlis, puis, après avoir reçu le canal vitellin, remonte jusqu'à l'utérus. L'ovaire massif s'étend dans le $1 / 3$ inférieur du proglottis. En coupe, il forme deux masses latérales en éventail d'acini très étroits. S'il n'a pas une section en $\mathrm{X}$ caractéristique, il présente néanmoins une tendance à cette allure (fig. 9).

L'utérus est constitué dans les jeunes segments par une masse médiane ventrale de cellules différenciées avec une lumière très étroite. Dans les proglottis âgés, il est sacciforme et occupe tout le 

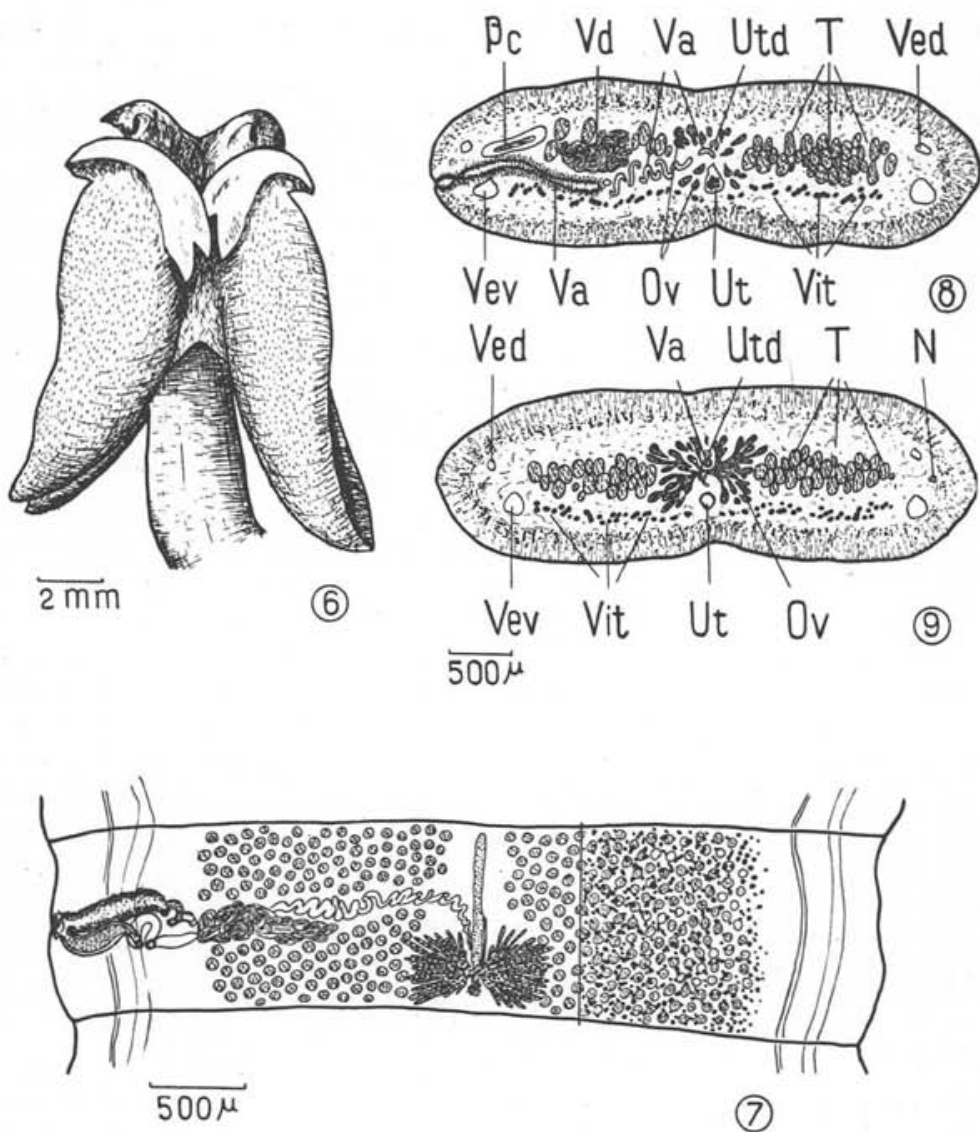

Fig. 6. - Gästrolecithus planus (Lintos, 1922). Scolex, vue latérale.

Fig. 7. - Gástrolecilhus planus (Linton, 1922). Anatomie vue ventrale semischématique. Les vitellogènes n'ont été représentés que sur le tiers latéral droit.

Fig. 8. - Gastrolecithus planus (Linton, 1922). Coupe transversale au niveau de la poche du cirre.

Fig. 9. - Gastrolecilhus planus (Livton, 1922). Coupe transversale au niveau de l'ovaire. N, nerf ; Ov, ovaire ; Pc, poche du cirre ; T, testicule ; Ut, utérus; Utd, utéroducte ; Va, vagin ; Ved, vaisseau excréteur dorsal ; Vev, vaisseau excréteur ventral ; Vd, vas deferens ; Vit, vitellogènes.

centre de l'anneau. La ligne médiane ventrale forme une zone de moindre résistance par où l'utérus se videra.

Les œufs dans l'eau de mer mesurent de 40 à $45 \mu$ de diamètre et contiennent un embryon hexacanthe de $20 \mu$. 
Les vitellogènes forment deux bandes de très petits follicules sur la face ventrale du proglottis.

Le système excréteur est représenté par quatre canaux longitudinaux (deux ventraux larges, deux dorsaux étroits).

REESIUM (1) PACIFERUM (Sproston 1948) Baylis 1950 (emend.)

Syn. Dinobothrium spinosum Baylis 1950.

Dinobothrium spinulosum Yamaguti 1952.

Dinobothrium humile Euzet 1952.

Matériel étudié :

Dinobothrium paciferum type British Museum.

Dinobothrium spinosum type British Museum.

Dinobothrium humile types Coll. Uni. Neuchâtel.

Dinobothrium spinosum Cabanes de Fleury, 12-5-1952.

Reesium paciferum, Cetorhinus maximus, Concarneau, 18-7-1938, 8-4-1943, 9-4-1943 (Coll. R.-Ph. Dollfus).

Lors de la description de $D$. humile, nous avons écrit que ce Cestode se séparait de $D$. paciferum, par la présence de quatre ventouses accessoires au lieu de huit chez l'espèce de Sproston, par le nombre inférieur de testicules et par l'allure des vitellogènes. Ayant eu connaissance, durant l'impression de la note sur $D$. humile, du travail de Baylis, nous avons assimilé notre espèce au $D$. spinosum de cet auteur. C'est sous ce nom que nous avons signalé ce parasite, le 12 mai 1952, avec C. Delamarre-Debouteville.

Depuis lors, nous avons pu examiner le matériel type de Dinobothrium paciferum. L'interprétation du scolex donnée par Sproston est erronée. Ce scolex porte quatre ventouses accessoires, une par bothridie, et huit pointes musculaires latérales aux extrémités du bourrelet qui surmonte la bothridie. Baylis, conscient de ces erreurs, a, malgré tout, créé l'espèce $D$. spinosum.

Après examen du matériel original, nous pensons que les deux espèces sont semblables et que l'espèce de Baylis est synonyme de D. paciferum. La description de Sproston étant complétée et amendée par celle de Baylis, Yamaguti a retrouvé ce parasite, comme nous induit en erreur par la description de Sproston, et, dans l'ignorance du travail de Baylis, l'a nommé $D$. spinulosum.

(1) Genre nouveau, dédié à Miss Gwendolen ReEs. 


\section{Morphologie}

Cestode anapolytique, dont la longueur varie entre 9 et $40 \mathrm{~mm}$., la largeur maxima entre $400 \mu$ et $1 \mathrm{~mm}$. Nous avons compté de 40 à 60 segments, dont les derniers (une dizaine) sont gravides.

Le scolex se compose de quatre bothridies sessiles, en forme de cuiller, étroites et allongées. Elles mesurent de $600 \mu$ à $1,2 \mathrm{~mm}$. de long et de 200 à $500 \mu$ de large. Leur dos est recouvert de petites épines de 4 à $8 \mu$ de long. Chaque bothridie est surmontée d'un bourrelet musculaire qui forme de chaque côté deux petites cornes charnues. La base de la corne latérale se prolonge sur la face dorsale par un lobe divisé en deux pointes (l'externe plus longue que l'interne).

Sur la crête, s'ouvre une ventouse accessoire de 130-160 $\mu$ de diamètre. C'est une ventouse typique complète, dont le bord interne est recouvert de quelques rangées de petites papilles à pointe mousse (fig. 10).

Le scolex est porté par un cou de 2 à $10 \mathrm{~mm}$., entièrement couvert d'épines aplaties, de 8 à $25 \mu$ de long (fig. 11).

Ces épines à base creuse sont semblables à celles qui existent chez Prosobothrium Cohn 1902, Phoreiobothrium Linton 1889, Platybothrium Linton 1890, Dicranobothrium Euzet 1953. Les épines diminuent de longueur et disparaissent dans les premiers anneaux.

Les segments, acraspèdes d'abord comme un simple trait transverse, s'allongent très rapidement. Le dernier segment, effilé à l'extrémité postérieure, mesure de 1,7 à $2,2 \mathrm{~mm}$. de long sur 0,42 à $0,60 \mathrm{~mm}$. de large.

Le pore sexuel alterne régulièrement et s'ouvre latéralement à la moitié du segment.

\section{Anatomie (fig. 12)}

Appareil mâle. - Les testicules, dont le nombre varie de 28 à 45 , sont situés entre l'ovaire et les vitellogènes. Le « vas deferens » forme un peloton serré à l'extrémité de la poche du cirre et entre celle-ci et la paroi du segment du côté poral. Cette poche, grande de $350-400 \mu$ sur $70-150 \mu$, s'étend obliquement vers le haut, un peu au-delà de la ligne médiane. Elle contient un long pénis armé d'épines de $10 \mu$ environ (fig. 13).

Appareil femelle. - L'ovaire est situé à la partie postérieure du segment. Il n'a pas en coupe l'allure en X, mais forme un double éventail de grands lobes joints par un isthme tranversal (fig. 14).

Le vagin s'ouvre dans un très court atrium génital, soit antérieurement, soit postérieurement, soit encore sur le même plan que la 
poche du cirre. Son trajet sinueux à grandes boucles l'amène ventralement jusqu'au niveau des conduits excréteurs antiporaux. Là, il descend, toujours en formant des boucles, vers le complexe ovarien.

L'utérus apparait d'abord comme une colonne de cellules différenciées à lumière très étroite. Bourré d'œufs, il s'étend latéralement et dorsalement. Il forme alors deux sacs, un antérieur et un postérieur, séparés par le vagin et la poche du cirre qu'il enveloppe.

Les vitellogènes sont groupés en deux bandes latérales, interrompues seulement au niveau de l'orifice génital.

Les œufs, petits et sphériques, leur diamètre varie entre 35 et $40 \mu$ (37-45 $\mu$, Yamaguti), possèdent un embryon hexacanthe de $20 \mu$ (22-27 $\mu$, Yamaguti).

Ce système excréteur est typiquement représenté dans le strobile par deux canaux ventraux larges et deux canaux dorsaux étroits.

Baylis a signalé une particularité du système musculaire. Le ver possède sur le milieu de la face dorsale deux faisceaux puissants de fibres longitudinales ; partout ailleurs, la couche des muscles longitudinaux est faible.

D'après ce qui précède, les caractères distinctifs des trois espèces sont les suivants :

\begin{tabular}{|c|c|c|c|}
\hline & $\begin{array}{c}\text { Dinobothrium } \\
\text { septaria } \\
\text { VAN BRNEDEN } 1889\end{array}$ & $\begin{array}{l}\text { Gastrolecithus } \\
\text { planus } \\
\text { (Linton 1922) }\end{array}$ & $\begin{array}{c}\text { Reesium } \\
\text { paciferun } \\
\text { (Sproston 1948) }\end{array}$ \\
\hline Hôte............ & $\begin{array}{c}\text { Lamna cornubica } \\
\text { Carcharhinus glaucus }\end{array}$ & Cetorhinus maximus & Cetorhinus maximus \\
\hline $\begin{array}{l}\text { Longueur...... } \\
\text { Scolex......... } \\
\text { Cou } \ldots \ldots \ldots \ldots \ldots\end{array}$ & $\begin{array}{c}70-110 \mathrm{~mm} \\
3-5 \times 8-10 \mathrm{~mm} \\
\text { Absent }\end{array}$ & $\begin{array}{c}200-825 \mathrm{~mm} . \\
6-8 \times 8-10 \mathrm{~mm} . \\
\text { Présent }\end{array}$ & $\begin{array}{l}\text { 9-40 } \mathrm{mm} . \\
0,6-2,2-\times 0,2-1,2 \\
\text { Présent, couvert } \\
\text { d'épines aplaties }\end{array}$ \\
\hline Testicules........ & $600-€ 50$ & $250-300$ & $28-45$ \\
\hline $\begin{array}{l}\text { Poche du cirre.. } \\
\text { Vagin } \ldots \ldots \ldots \ldots \ldots\end{array}$ & $\begin{array}{c}1,-1,4 \times 0,4-0,5 \mathrm{~mm} . \\
1 * \text { partie dirigée vers } \\
\text { l'avant avec } \\
2 \text { splineters }\end{array}$ & $\begin{array}{c}600-650 \mu \times 150-175 \mu \\
\text { Horizontal }\end{array}$ & $\begin{array}{c}350-400 \mu \times 70-150 \mu \\
\text { Irrégulier à grandes } \\
\text { boucles }\end{array}$ \\
\hline $\begin{array}{l}\text { Ovaire } \ldots \ldots \ldots \ldots \\
\text { Vitellogènes..... }\end{array}$ & $\begin{array}{l}\text { Section en } \mathrm{X} \\
\text { Latéraux }\end{array}$ & $\begin{array}{l}2 \text { masses en éventail } \\
\text { bandes ventrales. }\end{array}$ & $\begin{array}{c}2 \text { masses en éventail } \\
\text { Latéraux }\end{array}$ \\
\hline
\end{tabular}

\section{Formes larvaires}

Des formes larvaires se rapportant aux Dinobothrium ont été décrites par Linton et Dollfus. 


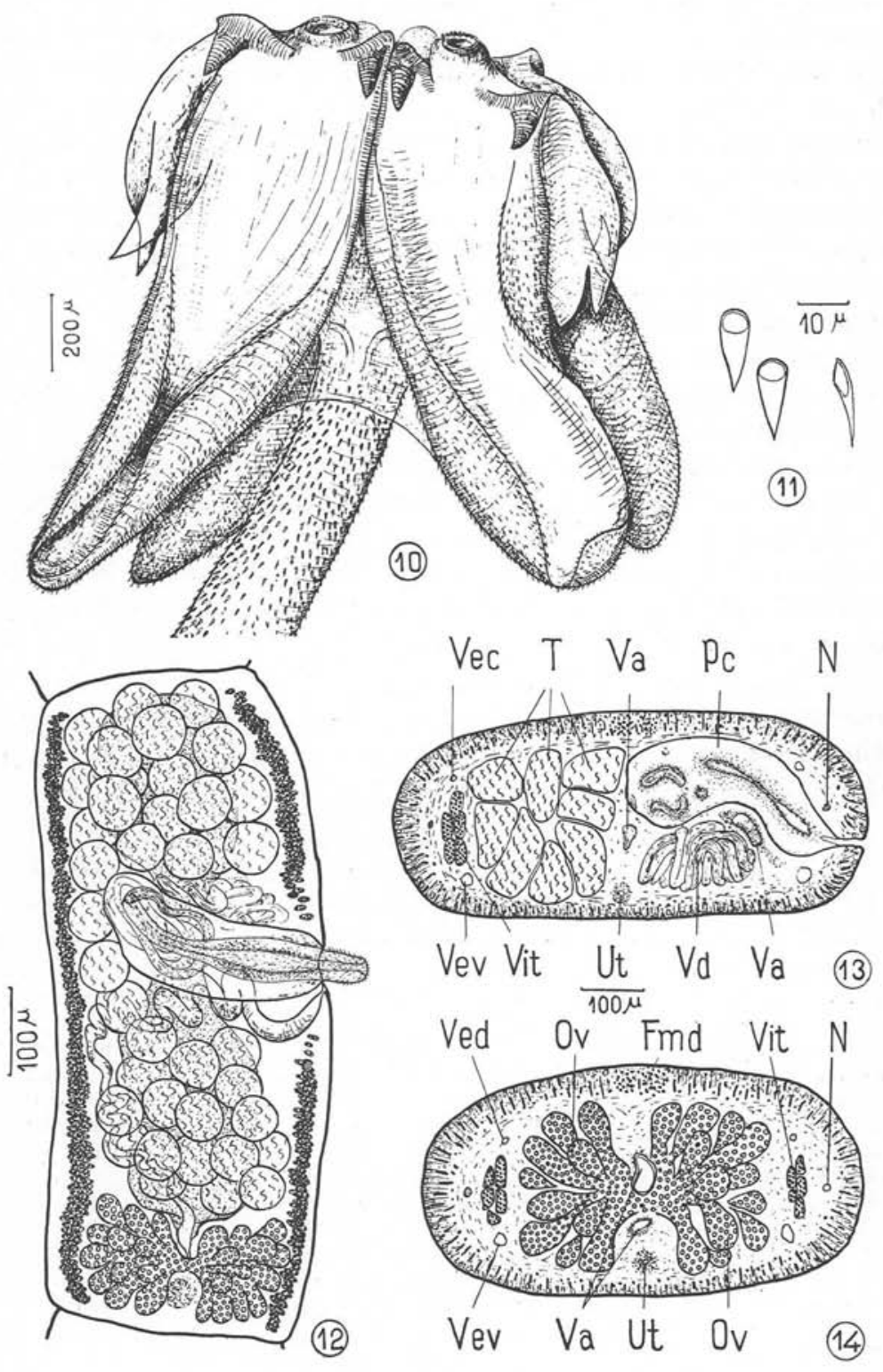

Fig. 10. - Reesium paciferum (Sproston, 1948). Scolex préparation totale.

Fig. 11. - Reesium paciferum (Sproston, 1948). Epines du cou, vues de face et de profil. 
Linton a, en 1897, attribué à un Thysanocephalum sp. une larve trouvée dans l'estomac d'un Céphalopode, Ommatrephes illecebrosus, à Woods Hole (Mass., U.S.A.).

Comme Dollfus, Sproston et Baylis l'ont fait remarquer, c'est à un Dinobothrium, Gastrolecithus ou Reesium, que doit être rapporté ce parasite.

Dollfus, en 1936, assimile à Dinobothrium plicitum une forme larvaire semblable à celle de Linton et récoltée à Concarneau, dans l'estomac d'un autre Céphalopode, Todaropsis eblanæ. Les bothridies de cette dernière larve mesuraient $500 \mu$ de long.

A quelle espèce doit-on rapporter ces formes larvaires ?

Les scolex de Dinobothrium, Gastrolecithus et Reesium varient énormément dans leur taille.

Pour D. keilini, Sproston avait noté un scolex de $8 \times 10 \mathrm{~mm}$., ce qui donne fixé $7 \times 9 \mathrm{~mm}$. Mais, avec cet adulte, il y a aussi deux jeunes formes de $1,12 \times 1,6 \mathrm{~mm}$. et $2,5 \times 3 \mathrm{~mm}$.

D. Guevarra a décrit sous le nom de D. plicitum de jeunes scolex qui mesuraient de 3 à $3,5 \mathrm{~mm}$. $\times 2,5$ à $3,5 \mathrm{~mm}$.

Nous avons de notre côté recueilli dans Cetorhinus maximus de nombreux Cestodes très variables dans leur taille et que nous avons rapportés à $D$. planum.

Nous notons, pour le scolex de six de ces parasites, les dimensions suivantes : $1,6 \times 2,3 \mathrm{~mm} .,-2,6 \times 3 \mathrm{~mm} .,-3 \times 2,8 \mathrm{~mm}$., $3 \times 3,4 \mathrm{~mm} .,-3 \times 3,95 \mathrm{~mm} .,-3 \times 4 \mathrm{~mm}$.

Dans l'ensemble, ils ont une taille voisine de celle des exemplaires de D. Guevarra, mais certains sont plus petits.

Ces jeunes individus correspondent-ils, comme nous l'avions vu, à Gastrolecithus planus? Le scolex ressemble à celui de Gastrolecithus, mais le strobile a une allure toute différente.

Les segments augmentent rapidement de taille, pour devenir aussi longs que larges, mais sans présenter d'ébauches d'organes génitaux. Ce strobile ressemble en petit à celui de Dinobothrium septa-

Suite des légendes des figures de la page 188

Fig. 12. - Reesium paciferum (Sproston, 1948). Préparation totale d'un segment sexué.

FIG. 13. - Reesium paciferum (Sproston, 1948).

Coupe transversale au niveau de la poche du cirre.

FiG. 14. - Reesium paciferum (Sproston, 1948). Coupe transversale au niveau de l'ovaire. Fmd, faisceaux musculaires dorsaux; $\mathrm{N}$, nerf; Ov, ovaire : Pc, poche du cirre ; T, testicules ; Ut, utérus ; Utd, utéroducte ; Va, vagin : Ved, vaisseau excréteur dorsal ; Vev, vaisseau excréteur ventral; Vd, vas deferens : Vit, vitellogènes. 
ria. Peut-être est-ce là des $D$. septaria qui, n'ayant pas rencontré leur hôte normal, ne peuvent achever leur croissance ? Cette hypothèse est cependant combattue par le fait que Cetorhinus a une nourriture, basée sur de petits crustacés planctoniques, différente de celle de Lamna cornubica, carnivore.

Le scolex doit s'accroître dans l'hôte définitif, car la tête des larves trouvées dans les Céphalopodes ont une taille nettement inférieure à celle du scolex de Dinobothrium septaria et Gastrolecithus planus. Ces larves se rapprocheraient plutôt des Reesium, mais là encore nous devons mettre en ligne de compte le régime de l'hôte. Comme l'écrit Baylis, « il est douteux que Cetorhinus puisse engloutir des Céphalopodes, à moins que ceux-ci ne soient extrêmement petits.

\section{Conclusions}

D'après les descriptions qui précèdent, nous voyons que les trois espèces de Dinobothrium qui ont un scolex extrêmement semblable ne possèdent pas la même anatomie.

D. septaria a celle d'un Phyllobothriidæ typique.

D. paciferum ressemble par les épines de la cuticule et la forme de son ovaire à des Prosobothriides.

D. planum, par la position ventrale de ses vitellogènes, se place complètement à part dans les Tetraphyllidea.

Nous proposons done :

$1^{\circ}$ De conserver le genre Dinobothrium Van Beneden 1889 avec l'espèce type, Dinobothrium septaria Van Beneden 1889, dans les Phyllobothriides, et plus particulièrement dans les Phyllobothriidæ, parmi les Phyllobothriinæ.

$2^{\circ}$ De séparer dans le nouveau genre Reesium le Dinobothrium paciferum Sproston 1948. Nous placerons cette espèce dans les Prosobothriides, à côté des Phoreiobothriidæ, dont ils se sépareront par l'absence de crochets à la partie antérieure des bothridies.

$3^{\circ}$ De conserver le genre Gastrolecithus Yamaguti 1952 pour le Dinobothrium planum Linton 1922, mais de séparer cette espèce dans une famille nouvelle que nous nommerons Gastrolecithidæ. Cette famille aberrante restera cependant dans les Tetraphyllidea.

Baylis, en 1926, a vu une parenté entre les genres Dinobothrium et Tetrabothrium.

Cette parenté est surtout visible dans le scolex. Il est certain que les coupes de la tête de Tetrabothrium diomedea telles que les donne 
Furhmann en 1931 ressemblent énormément à une coupe de scolex de Dinobothrium, Gastrolecithus ou Reesium.

Furhmann écrit d'ailleurs en 1932: «Le scolex des Tetrabothrium a une ressemblance marquée pour celui des Tétraphyllides. Ses organes de fixation rappellent par certains de leurs caractères des bothridies. En effet, ils ne possèdent pas, comme cela est caractéristique pour les Cyclophyllidea, de ventouses profondément enfoncées dans le parenchyme du scolex, mais des organes attachés à la surface de la tête, comme chez la plupart des Tétraphyllides. »

Chez Gastrolecithus, les vitellogènes, nous l'avons vu, forment deux bandes ventrales. Il est possible de concevoir la réduction de cette couche en une glande unique ventrale, mais située en avant de l'ovaire.

Furhmann écrivait encore en 1932 : « Les Tetrabothriidæ constituent la famille la plus primitive des Cyclophyllides et la seule dont la parenté possible qui pourrait être mise en avant devrait être recherchée chez les Tétraphyllides. »

A notre avis, Tétraphyllides et Tetrabothriidæ dérivent d'une souche commune. La famille des Gastrolecithidæ avec le genre Gastrolecithus, appartenant encore aux Tétraphyllides, formerait un terme de passage.

\section{RÉSUMÉ}

Le genre Dinobothrium, où l'on reconnaît seulement trois espèces valables, est divisé en trois genres.

Dinobothrium Van Beneden 1889, avec l'espèce Dinobothrium septaria Van Ben. 1889, est conservé dans la famille des Phyllobothriidæ.

Reesium n. g., avec l'espèce $R$. paciferum (Sproston 1948), est rangé parmi les Prosobothriides Euzet 1953.

Gastrolecithus Yamaguti 1952, avec l'espèce G. planus (Linton 1922), devient le type d'une famille nouvelle nommée Gastrolecithidæ, aberrante parmi les Tetraphyllidea.

\section{Bibliographie}

BuYLIS (H.-A.). - Some Tetrabothriid cestodes from whales of the genus Balanoptera. J. Linn. Soc. (Zool.), 36, 1926, pp. 161-172, fig. 1-6.

- A review of the species of Dinobothrium (Cestoda) with a description of a new species. Parasitology, 40, 1950, pp. 96-104, fig. 1-9.

Delamare (C.) et Euzet (L.). - Caractéristique d'un Squale Pèlerin Cetorhinus maximus (Gunner). Vie et Milieu, IV, 1952, fasc. 2.

Dollfus (R.-Ph.). - Cestodes des invertébrés marins et thalassoïdes (in Joyeux C. et BAER J. G. Faune de France, 30, 1936, pp. 503-539, fig. 541-569). 
Euzer (L.). - Sur deux Cestodes Tetraphyllides. Bull. Soc. Neuchat. Sc. Nat., 75, 1952, pp. 169-178, fig. 1-6.

- Suggestions pour une nouvelle classification des Cestodes Tétraphyllides. $X I V^{e}$ Congr. Int. Zool. Copenhague, 1953.

Funrmans (O.). - Cestoïdea. Kukenthal's Handb. Zool., 2, 1931, pp. 141-416, fig. 176-435.

- Les ténias des Oiseaux. Mém. Univ. Neuchâtel, 8, 1932, pp. 1-383, fig. 1-147.

Guevara Pozo (D.). - Cestodes del genero Dinobothrium parasitos de grandes Selacios pescados en las costas españolas. Rév. Iberica. Parasit., Lopez Neyra Mem. Vol., pp. 260-270, pl. I-II.

Joyetx (C.) et BAER (J.-G.). - Cestodes. Faune de France, n ${ }^{\circ} 30,1936$, pp. 1-613, fig. 1-569.

Linton (E.). - Notes on larval Cestodes Parasite of fishes. Proc. U.S. Nat. Mus., $20,1897, \mathrm{n}^{3} 1123$, pp. 787-824, pl. 61-68.

- A contribution to the anatomy of Dinobothrium a genus of Selachian Tapeworms ; with descriptions of two new species. Proc. U.S. Nat. Mus., 60, 1922, n० 2401 , pp. 1-13, pl. 1-4.

Lönnserg (E.). - Anatomische Studien über Skandinavische Cestoden. II. Zwei Parasiten aus Walfischen und zwei aus Lamna cornubica. Kgl. Sv. Vet. Handl., 24, 1892, n ${ }^{\circ} 16$, pp. 20-24.

- Ueber einige Cestoden aus dem Museum zu Bergen. Bergens Museum Aarbog, 4, 1898, pp. 1-23, pl. 1 .

Ması (L.). - Sulla presenza del Dinobothrium septaria in una Selache maxima. Bool. Soc. Zool. Ital., sér. 3, 1912, pp. 323.

Mota (P.). - Di alcune specie poco studiate o mal note di Cestodi. Ann. Mus. Zool. R. Univ. Napoli, Neseria, II, 1906, $\mathrm{n}^{\circ} 6$, pp. 1-12, pl. 3-4.

Nybelin (O.). - Notizen über Cestoden, II. Noch ein Fall von Dinobothrium septaria Van Ben. aus Selache maxima. Zool. Bidrag. Uppsala, vol. 3, 1914, pp. 228-230.

Perrenoud (W.). - Recherches anatomiques et histologiques sur quelques Cestodes de Selaciens. Rev. Suisse Zool., 38, 1931, pp. 469-555, fig. 1-50.

Scotr (Ph.). - Some notes on Fish parasites. 26th Ann. Rep. Fish. Bd. Scotl (For 1907), 1908, pp. 73.

Southwell (T.). - A monographe of the Tetraphyllidea with notes on related Cestodes. Mem. Liverpool. School. Trop. Med., N Séries 2, 1925, pp. 1368 , fig. 1-243.

Sproston (N. G.). - On the genus Dinobothrium Van Beneden (Cestoda) with a description of two new species from sharks and a note on Monorygma sp. from the electric ray. Parasitology, 39, 1948, pp. 73-90, fig. 1-25.

TSENG (S.). - Study on some Cestodes from fishes. Jor. Sci. Nat. Univ. Shantung. Tsingtao, 2, 1933, $\mathrm{n}^{\circ} 1$, pp. 1-21, fig. 1-28.

Van Beneden (P. J.). - Deux Cestodes nouveaux de Lamna cornubica. Bull. Ac. Roy. Belgique, 3, 17, pp. 1-9, pl. 1 .

Warde. (R. A.) et Mc Leod (J. A.). - The Zoology of Tapeworms. Univ. Minnes. Press, 1952, pp. 1-780, fig. 1-419.

Woodland (W. V. F.). - On Dinobothrium septaria VAN Beneden 1889 and Parabothrium bulbiferum Nybelin 1927. J. Parasitol., 13, 1927, pp. 231241, fig. 1-12.

YAmacitr (S.). - Studies on the helminth fauna of Japan. Part 49. Cestodes of fishes II. Acta Med. Okayama, 8, 1952, n 1, pp. 1-76, pl. 1-22. 